\title{
FENOLOGIA E PRODUTIVIDADE DO JAMBO (Syzygium malaccensis) NA AMAZÔNIA CENTRAL ${ }^{1}$
}

\author{
Martha de Aguiar FALCÃO ${ }^{2}$, Norival D. PARALUPPI ${ }^{3}$, Charles R. CLEMENT ${ }^{4}$
}

RESUMO - O jambeiro (Syzygium malaccensis Merr. \& Perry, Myrtaceae) é uma fruteira originária do sudeste da Ásia, hoje muito comum e apreciada na América do Sul e Central. O conhecimento de sua fenologia pode contribuir para o planejamento e o manejo do plantio, e a comercialização dos frutos. Num estudo realizado de janeiro de 1980 a dezembro de 1982 com árvores de cinco anos de idade, plantadas como ornamental em Manaus, AM, Brasil, constatouse que a floração e a frutificação do jambeiro ocorreram duas vezes ao ano: em meado da estação chuvosa (março) e durante o período de estiagem (julho-agosto). Ambos eventos foram rápidos, com duração de sete a 15 dias, levando cerca de um mes entre a floração e a safra. O estímulo climático à floração não foi evidente. O jambeiro apresentou vingamento moderado (4 a 10\%), como ocorre com a maioria das fruteiras da Amazônia. Um grande número de abelhas visitou as flores, sugerindo uma síndrome de polinização, em lugar de co-evolução com uma espécie ou gênero. A produtividade foi relativamente baixa, variando de 17,7 a $69,7 \mathrm{~kg} / \mathrm{planta}$, equivalente a 4 a 14 tha, sendo conveniente lembrar que essas árvores nunca foram adubadas.

Palavras-chave: floração, frutificação, vingamento, mudança foliar, insetos visitantes

\section{Phenology and Productivity of Malay Apple (Syzygium malaccensis) in Central Amazonia}

\begin{abstract}
The Malay apple (Syzygium malaccensis Merr. \& Perry, Myrtaceae) originated in southeast Asia, and is common and appreciated in South and Central America today. A knowledge of its phenology will help plan and manage the plantation and commercialization of its fruits. This study took place from January 1980 to December 1982 in 5-year-old trees planted as ornamentals in Manaus, AM, Brazil. Flowering and fruiting of Malay apple occurred twice a year, once in the mid-rainy season (March) and once in the mid-dry season (July-August); both events were very rapid, with durations of 7 to 15 days; the interval between flowering and harvest was about one month. The climatic stimulus for flowering was not evident. The Malay apple bad low fruit set (4 to 10\%), similar to the majority of Amazonian fruits. A large number of bee species visited the flowers, suggesting a pollination syndrom rather than recent local co-evolution with a single species or genus. Yield was relatively low, varying from 17.7 to $69.7 \mathrm{~kg} / \mathrm{plant}$ (4 to $14 \mathrm{t} /$ ha), although it must be remembered that these trees were never fertilized.
\end{abstract}

Key-words: flowering, fruiting, fruit set, leaf change, insect visitors

\section{INTRODUÇÃO}

O jambeiro
(Syzygium malaccensis Merr. \& Perry, Myrtaceae) é uma fruteira originária do sudeste da Ásia (Java, Sumatra e Península Malaysia), com distribuição pré-europeia ao sudoeste da Ásia e às ilhas do Pacífico (Panggabean, 1992). Hoje é comum e apreciado na América

'Pesquisa desenvolvida com apoio do convênio Universidade do Amazonas e Instituto Nacional de Pesquisas da Amazônia entre 1987 e 1990, e do Conselho Nacional de Desenvolvimento Científico e Tecnológico - CNPq entre 1997 e 1999.

${ }^{2}$ Bolsista do Programa de Capacitação Institucional do Instituto Nacional de Pesquisas da Amazônia - INPA, modalidade DTI, com apoio do CNPq. Av. Col. Teixeira, 386, Cond. Rio Tupana, Apto,602-A, 69030-481 Manaus, AM, Brasil. E-mail: mfalcao@internext.com.br

Instituto de Ciências Biológicas, Universidade do Amazonas, Av. Gal. R.O. Jordão Ramos, 3000 , 69077-000 Manaus, AM, Brasil.

${ }^{4}$ Instituto Nacional de Pesquisas da Amazônia - INPA, Cx. Postal 478, 69011-970 Manaus, AM, Brasil. 
do Sul e Central, onde é cultivado tanto como fruteira quanto como planta ornamental, devida a forma piramidal de sua copa que alcança $20 \mathrm{~m}$ de altura. A inflorescência contém de uma a doze flores, com pedícelos curtos. As flores são hermafroditas, actinomorfas, diclamideas, apopétalas ou dialipétalas, com numerosos estames vermelhos; ao cairem no solo formam um tapete vermelho, muito bonito. $\mathrm{O}$ fruto é drupáceo piriforme, vermelho a roxopurpúreo quando maduro, considerado como um dos maiores frutos do grupo Eugenia-Syzygium, com uma só semente, polpa espessa, branca, suculenta, de sabor acidulado, agradável, semelhante ao da maçã (Prance \& Silva, 1975; Cavalcante, 1991). Quanto a composição, $100 \mathrm{~g}$ de polpa do jambo contém $90 \mathrm{~g}$ de água, $0,3 \mathrm{~g}$ de proteína, $3,9 \mathrm{~g}$ de carboidratos, $1 \mathrm{~g}$ de fibras, 253 IU de vitamina $A, 0,1 \mathrm{mg}$ de vitamina C, e traços de vitamina B1 e B2 (Molesworth, 1967; Morton, 1987).

$\mathrm{O}$ jambeiro se adapta muito bem em climas com chuvas abundantes (Calzada Benza, 1980). As árvores são cultivadas em pomares, jardins e em ruas, por sua beleza, bem como por seus frutos que são apreciados pela população, sendo consumidos in natura, ou em forma de doces, compotas, geléias e refrescos. $\mathrm{Na}$ Indonésia os frutos são usados em saladas e são também conservados como "picles". Tanto as folhas como as raízes são usadas tradicionalmente na medicina caseira na Tailândia (Panggabean, 1992).

A fenologia estuda o efeito da periodicidade das condições climáticas, influenciado pelas condições edáficas e ecológicas em geral, sobre o ciclo biológico das plantas, especialmente sobre os orgãos reprodutivos e de crescimento vegetativo (Wielgolaski, 1974), O conhecimento da fenologia ajuda o planejamento e o manejo do plantio, e da comercialização dos frutos. Seguindo Prance \& Silva (1975) e Cavalcante (1991), o jambeiro floresce várias vêzes por ano, mas outras informações não existem na Amazônia. O objetivo deste trabalho foi observar as fases fenológicas e a produtividade do jambo durante três anos na Amazônia Central.

\section{MATERIAL E MÉTODOS}

O estudo foi realizado no período de janeiro de 1980 a dezembro de 1982, em uma área do Campo Experimental da Secretaria de Produção Agropecuária do Estado do Amazonas, no km 12 da Estrada Torquato Tapajós (AM-010 Manaus-Itacoatiara), Manaus, Amazonas. As árvores estudadas estavam com cinco anos de idade no início das observações e serviam como ornamentação da área.

O clima da região de Manaus é "Afi", no esquema de Köppen, com médias anuais de $2.478 \mathrm{~mm}$ de chuva e temperatura de $25,6^{\circ} \mathrm{C}$ (Ribeiro, 1976). Os dados climáticos usados (Fig.1A) foram obtidos da Estação Meteorológica do Ministério de Agricultura em Manaus, a $12 \mathrm{~km}$ da área de observação. O solo desta área é do tipo Latossolo Amarelo, de textura média (N. Falcão, INPA, com. pess., 2001). 
Para o estudo foram escolhidas dez árvores ao acaso; de cada árvore foram selecionados três ramos, sendo um grande, outro médio e um pequeno, para contagem das flores e dos frutos (Falcão, 1993). As observações foram realizadas semanalmente e a contagem das flores foi feita no período de floração máxima, devido ao curto período deste fenômeno, diferente da metodologia seguida em trabalhos anteriores (Falcão, 1993). Os frutos abortados foram contados debaixo de cada árvore.

Para estimar a produtividade de cada planta, foram escolhidos ao acaso vinte e cinco frutos maduros, que foram pesados e separados em polpa e semente; calculou-se a média e desvio padrão de cada variável. A casca não foi separada por ser uma película membranosa e consumida junto com a polpa.

$\mathrm{Na}$ época de floração máxima no ano de 1980, as observações foram realizadas diariamente das 5:30 às 19:00 h, com o objetivo de se observar e capturar os insetos visitantes. Os insetos capturados foram enviados a um especialista para identificação.

\section{RESULTADOS E DISCUSSÃO Floração}

Observou-se duas florações bem definidas: uma em março, na época chuvosa, e outra em julho ou agosto, durante o período de estiagem, aparentamente sem relação com as chuvas (Fig. 1B). A sincronia entre árvores foi sempre alta, variando menos de uma semana em cada lado da média da floração. O período de floração é muito curto, levando de 7 a 15 dias entre a abertura dos botões e a queda das pétalas ou das flores não polinizadas. Ochse et al. (1974) comentaram que o jambeiro floresce uma vez por ano na Ásia e várias vêzes por ano nos trópicos americanos, concordando com as observações aqui relatadas.

A maior floração foi a primeira,

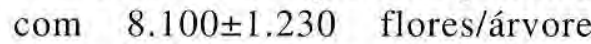
(variando de 5.500 a 9.600). Todas as florações subsequentes foram menores, não alcançando 5.500 flores/árvore. Supõe-se que esta redução foi devido ao pequeno estoque de nutrientes no solo, já que plantas ornamentais raramente são adubadas em áreas públicas.

\section{Frutificação}

A frutificação seguiu-se imediatamente após a floração (Fig. 1B), com a safra ocorrendo cerca de um mes após a floração máxima. Essas observações conflitam com o comentário de Cavalcante (1991) de que o jambo está presente nas feiras de Belém durante a maior parte do ano, embora Cavalcante salientou que existem dois períodos de maior abundância. Panggabean (1992) relatou que a maturação leva de 30 a 40 dias no sudeste da Ásia, similar ao observado aqui. Como ocorreu com a floração, a safra também teve duração curta, raramente excedendo a 15 dias. Também, o número de frutos produzidos na primeira safra foi maior (média de $840 \pm 280$ frutos/árvore; variando de 450 a 1270) que nas safras subsequentes, provavelmente pela mesma razão.

\section{Vingamento}

$O$ vingamento de frutos na primeira safra foi em torno de $10 \%$ das flores (Fig. 1C). Como ocorreu com a 
floração e a frutificação, o maior vingamento também foi o primeiro, com todos os vingamentos subsequentes menores, alcançando um mínimo (ao redor de 4\%) na época chuvosa do ano de 1982 . Supõe-se que este vingamento moderado seja parte de uma estratégia fisiológica chamada de 'ajustamento serial' (Lloyd, 1980; Browning, 1985), embora a falta de nutrientes no solo certamente contribuiu também. Este vingamento foi menor que observado em araçá-boi (Eugenia stipitata, 24\%) e camu-camu (Myrciaria dubia, 36\%), ambas Mirtáceas, mas maior que observado em cupuaçu (Theobroma grandiflorum, Sterculiacae; $0,9 \%$ ) na Amazônia central (Falcão, 1993).

\section{Mudança foliar}

A mudança foliar aconteceu sempre depois da safra, quando as folhas cairam parcialmente, como observado em diversas outras espécies frutíferas na Amazônia central (Falcão, 1993).

\section{Insetos visitantes}

Em abril de 1980, as árvores de jambeiro estavam todas floridas e cheias de insetos visitantes, na maioria
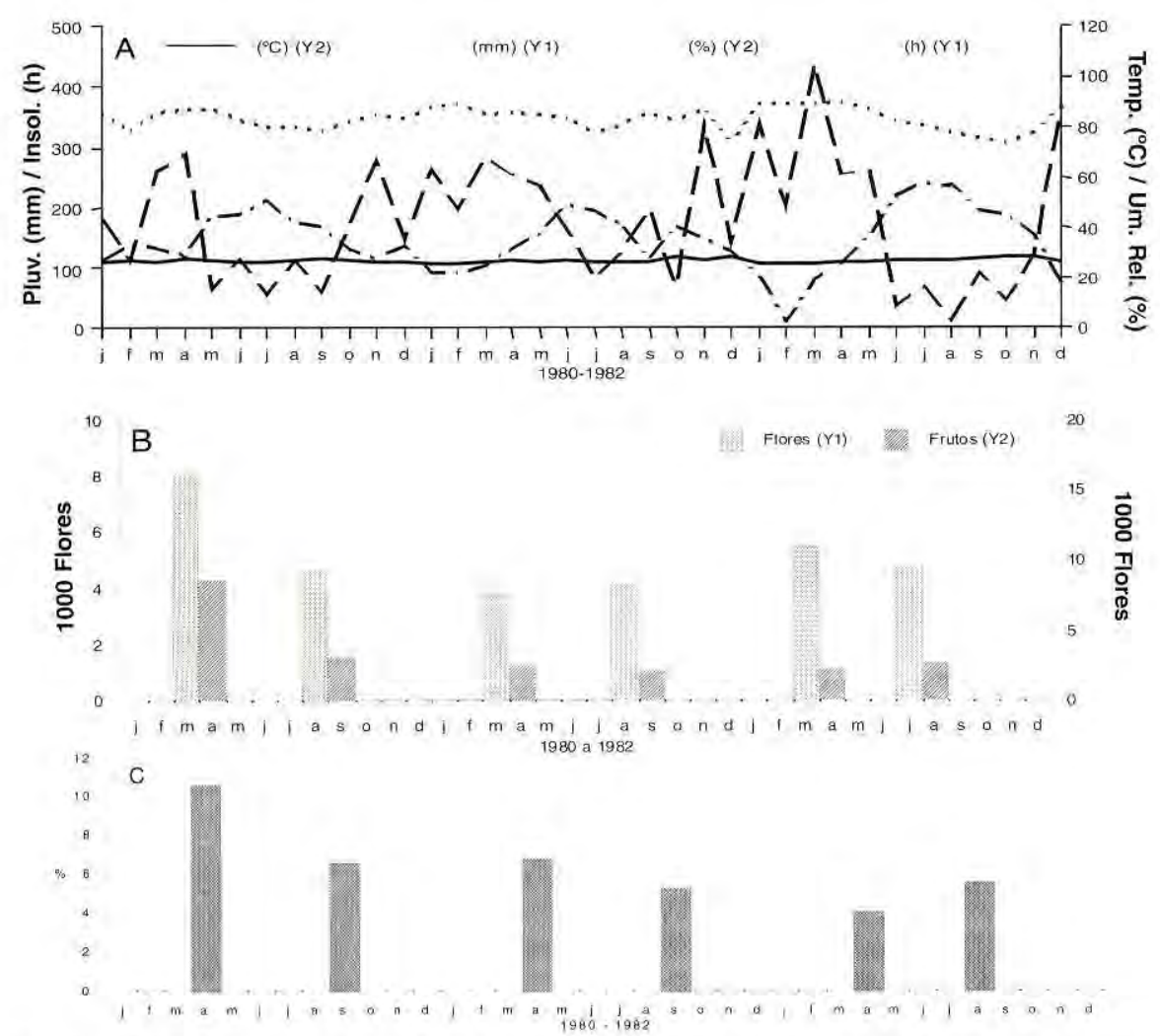

Figura 1. Fenologia do jambeiro (Syzygium malaccensis) na Amazônia Central de janeiro 1980 a dezembro 1982. A. Dados climatológicos de Manaus. B. Número de flores e frutos observados por mes. C. Percentagem de vingamento (frutos/flores). 
abelhas e vespas. Observou-se também beija-flores nas primeiras horas da manha e morcegos no início da noite. Entre as abelhas (Meliponini e Apini) foram encontradas: Trigona branneri Ck11.; T. williana Friese; $T$. dallatorreana Friese; Partamona pearsoni (Schwarz); P. mourei Camargo; $P$. testacea Klug; $P$. pseudomusarum Camargo; $P$. cf. nigrior Ckll.; Frieseomelitta savanensis Roubik; Frieseomelitta sp.; Apis mellifera L.; Melipona fulva Lep.; $M$. seminigra merrilae Ckll.; Plebea minima Gribodo; Duckeola ghilianii Spinola. Como ocorre em outras espécies frutíferas, não existe um visitante específico que possa ser caracterizado como o principal polinizador, haja vista que parece haver uma síndrome de visitantes, como observado para outras fruteiras da Amazônia (Falcão, 1993).

\section{Produtividade}

Os frutos dos dez jambeiros apresentaram peso médio de $80 \pm 7 \mathrm{~g}$ na primeira safra de 1980 , com $80 \pm 3 \%$ de polpa (mesocarpo + exocarpo) (Tab. 1). Com base no número de frutos nessa safra (847 por árvore), cada árvore produziu $69,7 \pm 23,6 \mathrm{~kg}$, dentro da variação de 20 a 85 kg/árvore relatada por Panggabean (1992). Usando um espaçamento de $6 \times 8 \mathrm{~m}$ (208 árvores/ha), a produtividade daquela safra foi ao redor de $14,5 \pm 4,9$ t/ha. No entanto, vale lembrar que estes cálculos são baseados na regra de três e que esta safra foi a maior observada. A safra menor ocorreu em setembro de 1981 e resultou na produção de apenas $17,7 \pm 6,8 \mathrm{~kg} /$ planta, equivalente a $3,7 \pm 1,4$ t/ha, similar à produtividade mínima relatado por Panggabean (1992).

\section{CONCLUSÕES}

A floração e a frutificação do jambeiro ocorreram duas vezes ao ano de 1980 a 1982, uma vez em meado da estação chuvosa (março) e uma vez durante o período de estiagem (julhoagosto), sendo que ambos eventos foram rápidos. Devido aos períodos de floração, o estímulo climático não foi evidente. O jambeiro apresentou vingamento moderado, embora um pouco menor que outras Mirtáceas estudadas na Amazônia central. Um

Table 1. Pesos médios ( \pm desvio padrão) dos frutos de jambo (Syzygium malaccensis) e seus componentes avaliados em 1980 na Amazônia Central.

\begin{tabular}{ccccc}
\hline Árvore & Frutos & Polpa & Sementes & $\%$ polpa \\
\hline 1 & $71,7 \pm 24,0$ & $58,8 \pm 20,2$ & $12,9 \pm 4,2$ & $81,7 \pm 3,0$ \\
2 & $89,3 \pm 11,3$ & $71,3 \pm 12,7$ & $15,4 \pm 4,5$ & $80,0 \pm 10,8$ \\
3 & $80,4 \pm 23,7$ & $62,1 \pm 24,8$ & $15,1 \pm 3,6$ & $77,0 \pm 16,6$ \\
4 & $80,2 \pm 16,1$ & $64,3 \pm 13,3$ & $15,9 \pm 4,5$ & $80,2 \pm 4,0$ \\
5 & $79,8 \pm 17,5$ & $61,4 \pm 18,8$ & $13,3 \pm 4,8$ & $78,3 \pm 17,1$ \\
6 & $93,1 \pm 21,7$ & $75,7 \pm 17,9$ & $17,4 \pm 5,5$ & $81,3 \pm 3,9$ \\
7 & $73,8 \pm 10,6$ & $61,4 \pm 8,5$ & $12,4 \pm 4,1$ & $83,4 \pm 4,1$ \\
8 & $74,7 \pm 12,1$ & $63,1 \pm 10,3$ & $11,6 \pm 4,0$ & $84,6 \pm 4,2$ \\
9 & $86,5 \pm 18,8$ & $73,3 \pm 17,3$ & $13,7 \pm 2,6$ & $84,4 \pm 2,8$ \\
10 & $92,3 \pm 15,2$ & $78,9 \pm 13,1$ & $13,3 \pm 4,1$ & $85,6 \pm 3,6$ \\
média & $82,2 \pm 7,3$ & $67,0 \pm 6,7$ & $14,1 \pm 1,7$ & $81,7 \pm 2,7$ \\
\hline
\end{tabular}


grande número de abelhas visitaram as flores, sugerindo um síndrome de polinização em lugar de co-evolução com uma espécie ou gênero. A produtividade foi relativamente baixa, embora similar aos relatos asiáticos.

\section{AGRADECIMENTOS}

Agradecemos ao Dr. João M.S. Camargo, da Faculdade de Medicina de Ribeirão Preto, SP, pela identificação das abelhas, à Dra. Beatriz R. Telles, do INPA, pela atualização e confirmação da nomenclatura das abelhas, à Dra. Marlene F. Silva, pela revisão do texto, às estagiárias biologas Maria José Barros e Janette Brito, da Universidade do Amazonas, pela ajuda na pesquisa de campo, e à bolsista do CNPq, Rozana Medeiros de Souza, da Universidade do Amazonas, pela colaboração no processamento dos dados.

\section{Bibliografia citada}

Browning, G. 1985. Reproductive behavior of fruit tree crops and its implications for the manipulation of fruit set. In: Cannel, M.G.; Jackson, J.E. (Eds.). Attributes of trees as crop plants. Inst. Terrestrial Ecology, NERC, Huntingdon, UK. pp. 409425 .

Calzada B., J. 1980. 143 frutales nativos. Universidad Nacional Agraria La Molina, Lima. 314p.

Cavalcante, P.B. 1991. Frutas comestiveis da Amazônia, 5 ed. Edições CEJUP, Belém. 279p.

Falcão, M.A. 1993. Aspectos fenológicos, ecológicos e de produtividade de algumas fruteiras cultivadas na Amazônia. Vol. II. Univ. do Amazonas, Manaus. 97p.
Lloyd, D.G. 1980. Sexual strategies in plants. I. An hypothesis of serial adjustment of maternal investment during one reproductive season. New Phytologist, 86:69-79.

Molesworth, A.B. 1967. Malayan fruits. Donald Moor Press, Singapore. pp. 115 125.

Morton, J. 1987. Fruits of warm climates. Morton Collectanea, University of Miami, Coral Gables, FL. pp. 378-383.

Ochse, J.J.; Soule, Jr., M.J.; Dijkman, M.J.; Wehlburg, C. 1974. Cultivo y mejoramiento de plantas tropicales y subtropicales. Volume I. Editorial Limusa, México. pp. 378-383.

Panggabean, G. 1992. Syzygium malaccense (L.) Merr. \& Perry. In: Verheij, E.W.N.; Coronel, R.E. (Eds.) Plant Resources of South-East Asia, No. 2: Edible Fruits and Nuts. Prosea, Bogor, Indonesia. pp. 292294.

Prance, G.T.; Silva, M.F. 1975. Árvores de Manaus. Instituto Nacional de Pesquisas da Amazônia, Manaus. 312p.

Ribeiro, M. N. G. 1976. Aspectos climatológicos de Manaus. Acta Amazonica, 8 (2) : 229-233.

Wielgolaski, F.E. 1974. Phenology in agriculture. In: Lieth, H. (Ed.). Phenology and seasonality modeling. Chapman \& Hall, London. pp.369-381. 